\title{
Non-invasive evaluation of electrical cardiac injury
}

\author{
RUBEN F LEWIN, ALEX ARDITTI, SAMUEL SCLAROVSKY \\ From the Israel and Ione Massada Center for Heart Disease, Beilinson Medical Center, Petah Tiqva, Israel
}

SUMMARY Non-invasive study of a 19 year old man, who sustained severe electric current injury, showed electrocardiographic and enzymatic changes simulating acute myocardial infarction. Serial echocardiographic examinations showed reversible global left ventricular dysfunction attributed to extensive myocardial injury which resolved within a few days. Clinical, electrocardiographic, and echocardiographic follow-up, after one year, showed no cardiac abnormalities.

Tachycardia, ST-T changes, arrhythmias, and conduction disturbances are well known cardiac complications in patients surviving injuries by electrical current. ${ }^{12}$ Non-invasive cardiac evaluation has seldom been reported. ${ }^{3}$

We describe a patient with electrocardiographic and enzymatic changes simulating myocardial infarction in whom serial echocardiograms disclosed reversible global left ventricular dysfunction.

\section{Case report}

A 19 year old man was admitted as an emergency approximately 15 minutes after suffering electric shock by 220 volts $\mathrm{AC}$ at $50 \mathrm{~Hz}$, during manipulation of a home generator. The patient lost consciousness immediately and was given cardiopulmonary resuscitation by his relatives until his arrival at the hospital. On admission, cardiac arrest was seen on the monitor. Resuscitation treatment, including intracardiac adrenaline $0.1 \mathrm{mg}$ and calcium gluconate $10 \% 10 \mathrm{ml}$, was administered, resulting in ventricular fibrillation. With cardioversion the patient developed idioventricular rhythm which converted immediately to sinus rhythm. A 12 lead electrocardiogram suggesting myocardial injury was recorded (Fig. 1A).

On admission, no signs of entry or exit of electrical injury were found on the skin. A systolic and diastolic

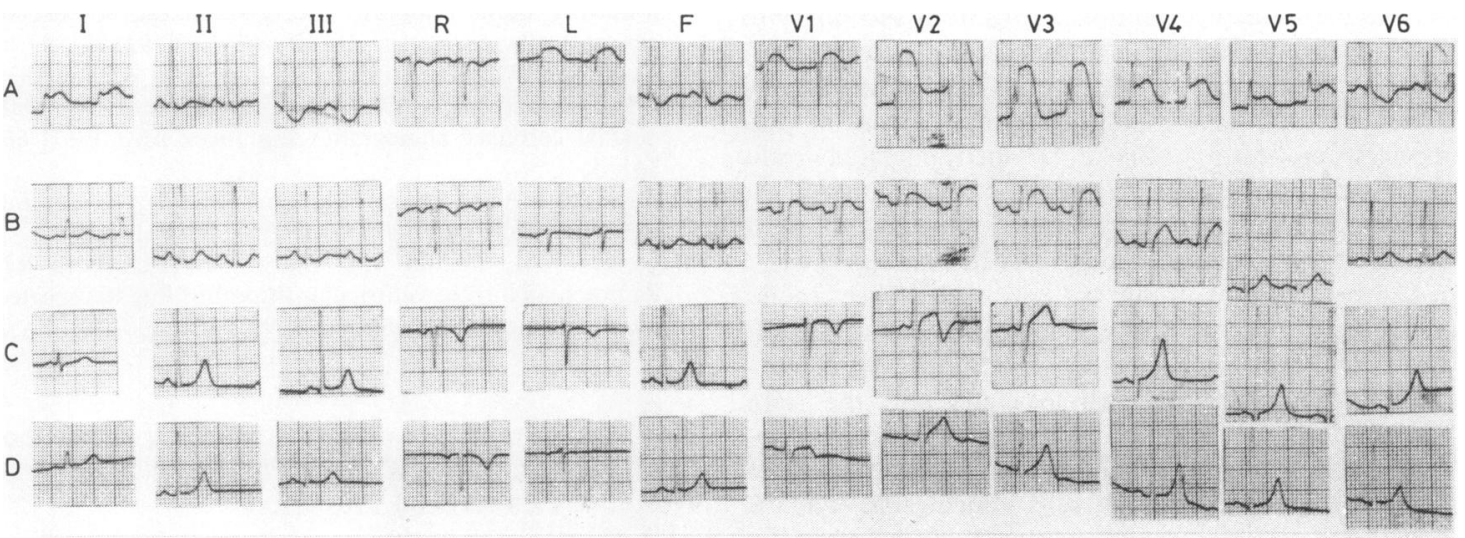

Fig. 1 Serial electrocardiographic changes in electrocution. (A) On admission ( 30 minutes after electrical injury) ST segment elevation in leads I, aVL, VI-5; ST segment depression in III, aVF, V6. (B) 24 hours after admission lower degree of ST segment elevation in I, VI-4. No ST depression is seen. (C) Three days after admission ST elevation in VI-3. Inverted T waves in aVL, VI-2. Peaked T waves in II, III, aVF, V4-6. (D) Six months after admission-normal electrocardiogram. 


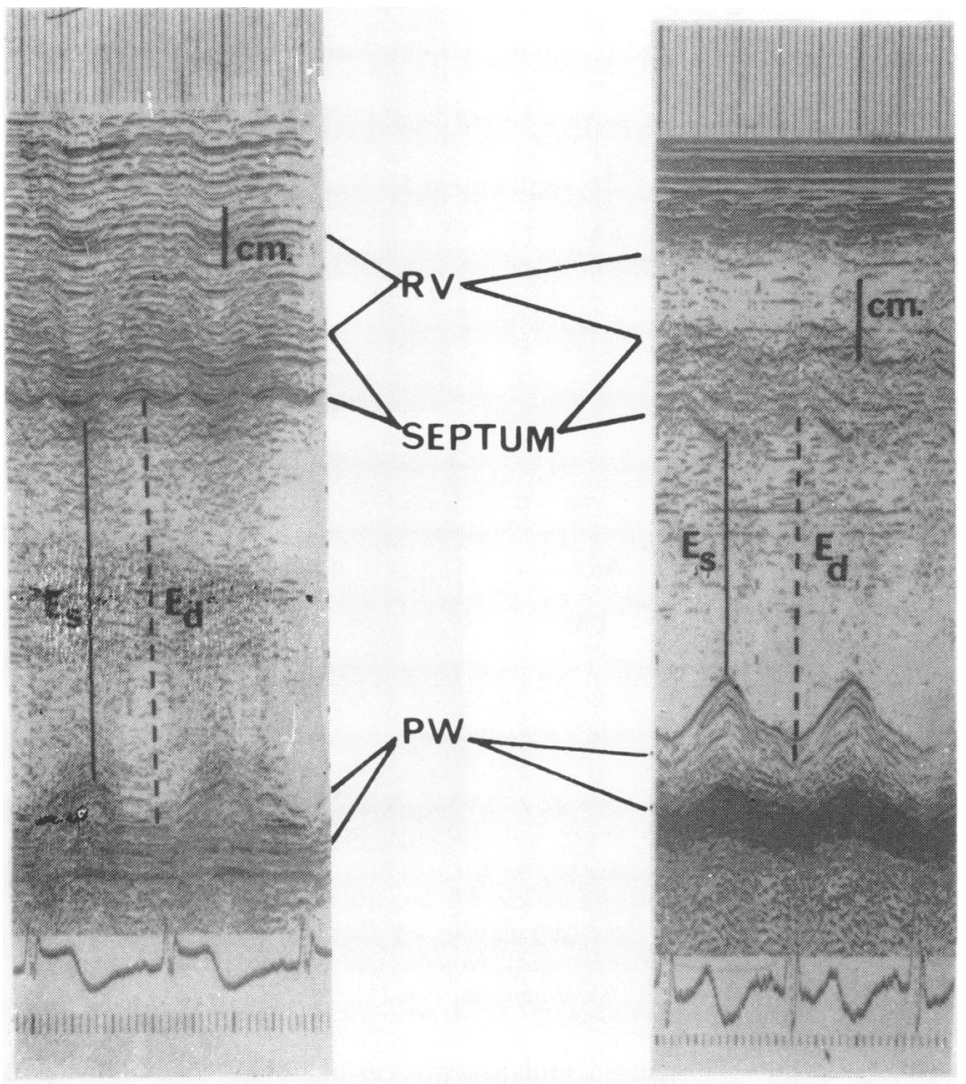

Fig. 2 Comparison of $M$-mode echocardiographic changes. On admission (left side) $\rightarrow$ right ventricular end-diastolic dimension, $2.5 \mathrm{~cm}$. Septal thickness, $0.8 \mathrm{~cm}$. Left ventricular end-diastolic dimension $(E d), 7.8 \mathrm{~cm}$, left ventricular end-systolic dimension (Es), $6.5 \mathrm{~cm}$. Percentage of fractional shortening: 19. Three days after admission (right side). Right ventricular end-diastolic dimension, $1.2 \mathrm{~cm}$. Septal thickness, $1.1 \mathrm{~cm}$. Left ventricular end-diastolic dimension $(E d), 5 \cdot 8 \mathrm{~cm}$. Left ventricular end-systolic dimension $(E S), 3.3 \mathrm{~cm}$. Fractional shortening, $43 \%$. The posterior wall thickness $(P W)$ was similar in the two examinations $(0.8 \mathrm{~cm})$. The improvement in septal and posterior wall motion between the two echocardiographic examinations is noted. $R V$, right ventricle; $\mathrm{cm}, 1 \mathrm{~cm}$.

pericardial friction rub was heard. The rest of the physical examination was normal. Cardiac enzymes on admission were raised (AST 94 (normal 40); LDH 403 (normal 225); CK $1310 \mathrm{IU} / \mathrm{ml}$ (normal $85 \mathrm{IU} / \mathrm{ml}$ ), with $8 \%$ MB CK (normal 0 )). Other laboratory tests were normal. At this stage an M-mode echocardiographic examination showed a left ventricular end-diastolic dimension of $7.8 \mathrm{~cm}$ and left ventricular end-systolic dimension of $6.5 \mathrm{~cm}$ (fractional shortening $=19 \%$ ) (Fig. 2). On two dimensional echocardiography global hypokinesis was recorded (Fig. 3). No evidence of pericardial effusion was recorded in this or subsequent echocardiographic studies. Three days after admission, the electrocardiographic changes improved (Fig. 1C) and the next echocardiographic examination showed that the end-diastolic dimension was $5.8 \mathrm{~cm}$ and the end-systolic dimension was $3.3 \mathrm{~cm}$ (fractional shortening 43\%) (Fig. 2). Comparing the two dimensional echocardiographic examination performed at the time of admission and three days later, thickening of the myocardial muscle was noted. Global left ventricular contraction had improved significantly (Fig. 3). CK at this stage was $1083 \mathrm{IU} / \mathrm{ml}$ with $5 \% \mathrm{MB}$ CK. A radio- isotopic cardiodynamic study was performed showing ejection fraction of $69 \%$, with normal regional wall motion. One year after discharge the electrocardiogram and the echocardiographic examination were found to be normal and the patient was free of any sign or symptom of cardiac damage.

\section{Discussion}

Cardiac injury after electrical current shock has seldom been reported in detail.1-3 Lightning stroke was reported to be associated with cardiac injury most often diagnosed by cardiac enzymes (CK, AST, LDH) and serial electrocardiographic changes. ${ }^{3-5}$ In most of the necropsies in these cases, myocardial necrosis has been seen. ${ }^{6}$

Whether or not electrocution was the sole contributor to the electrocardiographic, enzymatic, and echocardiographic changes in our case is a difficult question. Other causes of ST segment elevation such as pericardial injury were not supported because of the absence of pericardial fluid and the appearance of a pericardial friction rub only briefly after DC cardio- 


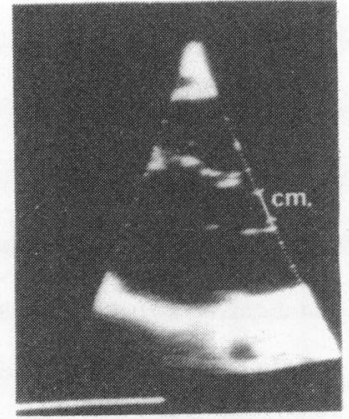

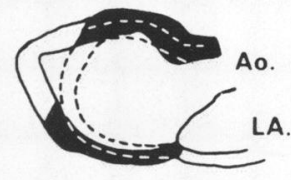

I L. Axis

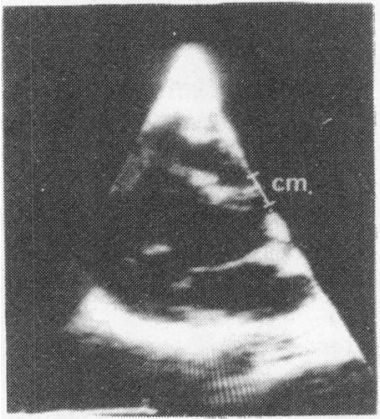

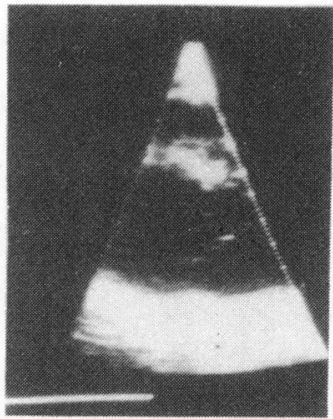

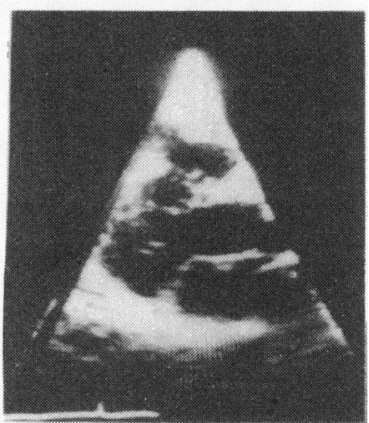

Fig. 3 Two dimensional echocardiographic long axis view of the left ventricle. The two upper figures-on admission, the two lower figures-three days later. On the left side of the figure diastolic stop-frames, on the right side of the figure systolic stop-frames. The middle diagram shows the thickness and contraction sequential changes. $I: L$. axis-on admission; II: L. axis - three days later; Ao, aorta; LA, left atrium; $\mathrm{cm}, 1 \mathrm{~cm}$.

version. Myocardial contusion during cardiopulmonary resuscitation with subsequent haematoma or coronary artery laceration cannot be ruled out definitely; it seems, however, to be a rare complication of cardiac resuscitation.

The resolution of the electrocardiographic abnormalities seen in our case supports the hypothesis that a "patchy" necrosis is present in the myocardium and the electrocardiographic localisation of signs of injury may be fortuitous. Transient ST segment elevation after elective DC cardioversion not followed by concomitant rise in MB CK or appropriate $\mathrm{LDH}$ isoenzymes or myocardial scintigraphy abnormalities has been reported. ${ }^{7}$ From the enzymatic point of view, it is very probable that myocardial injury occurred; but myocardial infarction cannot be proven. Two dimensional echocardiography showed a global left ventricular hypokinesis (Fig. 3), especially when it was compared with the echocardiographic examination performed three days later. The percentage of fractional shortening improved from 19 to 43 (Fig. 2) in three days, together with electrocardiographic normalisation of ST segment elevation (Fig. 1) and fall of $\mathrm{MB} C \mathrm{CK}$ in the serum from $8 \%$ to $5 \%$ of the total CK activity.

It is suggested that electrical injury produces myocardial damage, with resultant electrocardiographic tracings simulating myocardial infarction and high $\mathrm{MB} \overrightarrow{\overrightarrow{0}}$ $\mathrm{CK}$ levels, which resolves in a few days. This is supported by the echocardiographic findings of left ventricular hypokinesis reversing after three days.

\section{References}

1 DiVincenti FC, Moncrief JA, Pruitt BA, Jr. Electrical injuries: a review of 65 cases. $\mathcal{F}$ Trauma 1969; 9: 497-507. 8

2 Baxter CR. Present concepts in the management of major electrical injury. Surg Clin North Am 1970; 50: 1401-18.

3 Jackson SHD, Parry DJ. Lightning and the heart. Br Heart f 1980; 43: 454-7.

4 Hanson GL, McIlwraith GR. Lightning injury: two case histories and a review of management. BrMed $\mathcal{F}$ 1973; iv: N $271-4$.

5 Myers GJ, Colgan MT, Van Dyke DH. Lightning-strike $\mathcal{O}^{-}$ disaster among children. $\mathcal{F} A M A$ 1977; 238: 1045-6.

$6 \mathrm{Kravitz} \mathrm{H}$, Wasserman MJ, Valaitis J, Anzinger RE, $\mathrm{O}$ Naidu SH. Lightning injury. Am $\mathcal{J}$ Dis Child 1977; 131 413-5.

7 Chun PKC, Davia JE, Donohue DJ. ST-segment elevationo with elective DC cardioversion. Circulation 1981; 63: $220-4$.

Requests for reprints to Dr S Sclarovsky, Massada Center for Heart Diseases, Beilinson Medical Center, $\stackrel{\mathbb{Q}}{\mathbb{Q}}$ Petah Tiqva 49 100, Israel. 